\title{
Mineração
}

\section{Avaliação do efeito da granulometria no processo de flotação}

\author{
(Study of the effect of particle size on flotation process)
}

\author{
Neymayer Pereira Lima \\ Engenheiro de Minas, Engenheiro de Processo Sênior, CVRD \\ E-mail:neymayer.lima@vale.com \\ Geoge Eduardo Valadão \\ Engenheiro de Minas, Professor Associado, UFMG \\ E-mail:gvaladao@demin.ufmg.br
}

\section{Resumo}

A flotação é um dos principais processos de concentração, sendo empregado para diversas classes de minerais (sulfetos, óxidos, silicatos, fosfatos, etc.), como também para uma variedade de tamanhos de partículas. Estudos anteriores mostraram que a flotação pode ser empregada com sucesso para faixas granulométricas entre 10 e $300 \mu \mathrm{m}$. A seletividade do processo de flotação torna-se muito baixa fora desses limites granulométricos, porque as condições hidrodinâmicas do sistema são incapazes de manter o nível de flotabilidade ideal das partículas. Diferentes tamanhos de partículas são encontrados nos atuais sistemas de flotação, que podem reduzir a seletividade do processo, devido às diferenças de comportamento. Esse trabalho avaliou a flotação em bancada das frações $-150+45 \mu \mathrm{m}$ e $-45 \mu \mathrm{m}$ em comparação com a fração global $-150 \mu \mathrm{m}$ de um minério de ferro itabirítico, sob diferentes condições de $\mathrm{pH}$, dosagens de reagentes e rotação de condicionamento. Os resultados obtidos mostram que essas frações apresentam diferentes comportamentos no processo de flotação, indicando que é possível aumentar a recuperação em massa e a seletividade, quando essas frações são flotadas separadamente.

\begin{abstract}
Flotation is one of the main concentration processes and has been employed for many kinds of minerals (sulphides, oxides, silicates, phosphates, etc), and also for different particle sizes. Early studies showed that flotation can be successfully performed for particle sizes from 10 to $300 \mu \mathrm{m}$. Flotation selectivity becomes very low outside these size limits, because the hydrodynamic conditions can't maintain the ideal flotability levelfor the particles. Different particle sizes are found in the present-day flotation machines, which can reduce the process selectivity due to their different behaviors. This work aimed to evaluate the laboratory flotation studies of three particle sizes $(-150 \mu \mathrm{m},-150+45 \mu \mathrm{m}$ and $-45 \mu \mathrm{m})$ of an itabirite iron ore under different conditions of $\mathrm{pH}$, reagent dosage, and conditioning rotation. The obtained results show that these size fractions have different behaviors in the flotation process, indicating that it is possible to increase the mass recovery and the selectivity when these fractions are separately flotted.
\end{abstract}

Keywords: Size, flotation, selectivity.

Palavras-chave: Granulometria, flotação, seletividade. 


\section{Introdução}

A flotação é um dos principais processos de concentração, tendo sido empregada há aproximadamente cem anos para uma ampla classe de minerais: sulfetos, óxidos, fosfatos, silicatos, carvão e sais solúveis. O desenvolvimento da indústria mineral foi muito influenciado a partir da descoberta e utilização da flotação. Os processos físicos tradicionais, gravíticos, magnéticos e eletrostáticos, não teriam permitido a escala de produção necessária aos metais básicos. Não teria sido possível a produção atual dos metais nobres, nem a produção de fertilizantes fosfatados necessários ao desenvolvimento da agricultura. Parte da produção mundial de minérios de ferro, necessária à produção de aço nos níveis de consumo atual, só tornou-se possível nas últimas décadas com a utilização do processo de flotação em larga escala [1].

Os processos de flotação de minérios de ferro, seja através de células mecânicas ou de colunas, são alimentados por partículas com tamanhos entre 10 e $150 \mu \mathrm{m}$, sendo removidas as partículas ultrafinas (lamas) através de ciclonagem por afetarem negativamente o processo e o “top size” limitado entre 5 a $10 \%$ acima de $150 \mu \mathrm{m}$. Esse amplo gradiente de tamanhos de partículas pode comprometer a seletividade do processo, dadas suas possíveis diferenças de comportamento (hidrofobicidade, área superficial específica, peso, etc).

Alguns autores [2,3] mostram o mecanismo de flotação, através de conceitos probabilísticos, pela expressão:

$\mathrm{Pf}=\mathrm{Pc} \times \mathrm{Pa} \times \mathrm{Ps}$

onde:

$\mathrm{Pf}=$ probabilidade de flotação.

Pc $=$ probabilidade de colisão entre as partículas.

$\mathrm{Pa}=$ probabilidade de adesão (adelgaçamento e ruptura do filme líquido durante a colisão).

Ps = probabilidade de formação de um agregado estável, capaz de suportar as turbulências no interior das máquinas de flotação.
A probabilidade de colisão mereceu a atenção de diversos pesquisadores (Reay, 1975, Flint, 1971, Collins \& Jameson, 1976 e Anfruns \& Kitchener, 1977), que mostraram que Pc está diretamente relacionada com variáveis físicas, tais como: densidade, viscosidade da polpa, velocidade relativa bolha-partícula e, em particular, com os diâmetros da partícula e da bolha. Uma expressão primeiramente deduzida (Collins \& Jameson, 1976) mostra que, para uma dada vazão de gás, a eficiência de colilsão (Ec) seria dada por:

$\mathrm{Ec} \approx(\mathrm{dp} / \mathrm{db})^{2}$

onde:

dp = diâmetro da partícula

db = diâmetro da bolha

Essa expressão está de acordo com resultados experimentais obtidos por Collins e Jameson (1976) e Ray e Ratcliff (1975), que encontraram uma correlação:

$\mathrm{Ec} \approx \mathrm{dp}{ }^{1,5}$

Segundo Oliveira (2006), é bastante difundida, na literatura técnica, a concepção de que o rápido e desproporcional consumo de coletor pelas partículas finas, devido à sua maior área superficial específica, acarreta uma menor cobertura hidrofóbica na superfície das partículas grossas, que seriam, por essa razão, menos flotáveis. Essa concepção foi respaldada inicialmente pelos trabalhos experimentais de Robinson (1975), referente ao sistema quartzo-dodecilamina, e de Glembotsky (1968), referente ao sistema pirita-xantato. Esses autores observaram que uma maior concentração de reagentes era necessária para flotar partículas maiores.

A eficiência de flotação de partículas finas foi objeto de estudos mostrados por Trahar (1981) e Schulze (1984), que demonstraram que a dificuldade de recuperação dessas partículas pode ser atribuída a fatores hidrodinâmicos e ao efeito da carga elétrica das partículas e das bolhas.

Algumas alternativas foram propostas visando ao aumento na recuperação de finos, com base na agregação de partículas minerais por intermédio de reagentes ou da interação com outras partículas minerais. Tais alternativas são a flotação transportadora e a flotação por cisalhamento. O processo de floculação por cisalhamento objetiva à formação de agregados seletivos de partículas hidrofobizadas em um sistema turbulento e posterior flotação desses agregados. Como resultado da agitação turbulenta e das colisões, ocorre a formação desses agregados através do efeito hidrofóbico Brum et alii (1995). Bulatovic e Salter (1989) verificaram que a flotação, após condicionamento com alta agitação, mostrou aumento na recuperação global das partículas portadoras de cobre. Estudos realizados (Brum et alii, 1995) mostram que o condicionamento turbulento, como tratamento da polpa prévio a flotação, aumentou a recuperação de finos de ouro e sulfetos de cobre. Para o caso do ouro, a cinética de flotação aumentou, em 7 vezes, a recuperação em $24 \%$ e o teor em $50 \%$ no concentrado. Esses resultados foram interpretados pelo aumento da concentração de partículas hidrofóbicas finas e ultrafinas, como resultado do processo de agregação por cisalhamento seguido pela flotação transportadora.

\section{Material e métodos}

A amostra utilizada ness e trabalho representa um minério de ferro itabirítico proveniente do Quadrilátero Ferrífero. A caracterização foi feita através de análise química (global e por faixa), análise mineralógica (microscópio ótico de luz refletida) e análise granulométrica.

A Figura 1 mostra o fluxograma de obtenção e preparação da amostra.

Os testes de flotação foram realizados segundo planejamento de experimentos em dois níveis (mínimo e máximo) e quatro variáveis (dosagens de amido e amina, pH e rotação durante condicionamento). As Tabelas 1, 2 e 3 mostram os níveis destas variáveis para as três frações granulométricas avaliadas:

\section{Resultados e discussão}

A Tabela 4 mostra a análise granuloquímica da amostra estudada. 
Neymayer Pereira Lima et al.

As Figuras 2, 3, 4 e 5 mostram os resultados de recuperação em massa e teor de $\mathrm{SiO}_{2}$ no concentrado para as diferentes condições de reagentes (amina e amido), pH e rotação durante condicionamento. Conforme observado, as três frações granulométricas apresentam diferentes dosagens de amina para obtenção de concentrados com teores de $\mathrm{SiO}_{2}$ abaixo de $0,80 \%$. Os melhores resultados foram obtidos para uma dosagem de amido de $1000 \mathrm{~g} / \mathrm{t}_{\text {alim }}$ (g/t de alimentação) para todas as frações. A fração global $(-150 \mu \mathrm{m})$ apresenta os melhores resultados em $\mathrm{pH}$ 9,5, enquanto as outras duas em pH 10,5. O aumento da rotação durante condicionamento, de 1400 para $2000 \mathrm{rpm}$, possibilitou pequeno ganho de qualidade no concentrado da fração global, sem interferir na recuperação em massa. Para as demais frações, houve ligeiro aumento no teor de $\mathrm{SiO}_{2}$ nos concentrados.

A Figura 6 mostra o desempenho comparativo na flotação para as frações $-150 \mu \mathrm{m}$ global e $-150 \mu \mathrm{m}$ fracionado $(-150+45 \mu \mathrm{m}$ e $-45 \mu \mathrm{m}$ flotadas separadamente), indicando a possibilidade de aumento na recuperação metálica, quando essas frações são flotadas separadamente. Para obtenção desses resultados, a fração $-150+45 \mu \mathrm{m}$ indica a necessidade de maior dosagem de coletor, conforme mostrado na Figura 7.

\section{Considerações finais}

Os resultados obtidos mostram que as frações $-150 \mu \mathrm{m}$ (global), $-150+45 \mu \mathrm{m}$ e -45 $\mu \mathrm{m}$ apresentam diferentes comportamentos no processo de flotação em bancada.

Para obtenção de concentrados com teores de $\mathrm{SiO}_{2}$ abaixo de 0,80\%, são necessários diferentes dosagens de amina e diferentes valores de $\mathrm{pH}$.

Os resultados mostram a possibilidade de aumento de recuperação em massa, mantendo o limite de especificação de concentrado, quando as frações $-150+45 \mu \mathrm{m}$ e $-45 \mu \mathrm{m}$ são submetidas separadamente ao processo de flotação.

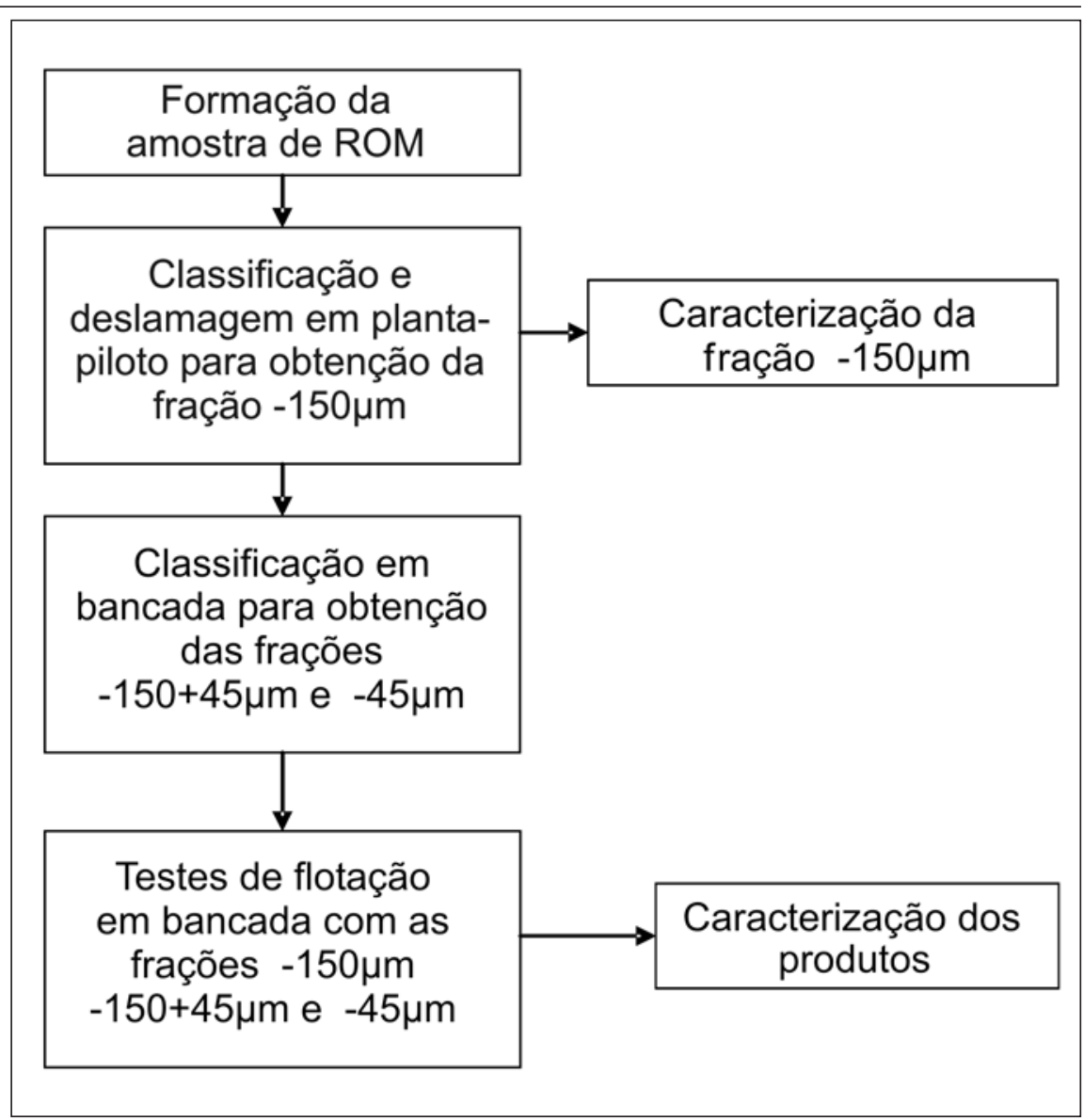

Figura 1 - Fluxograma de obtenção e preparação da amostra.

Tabela 1 - Níveis das variáveis (fração -150 $\mu \mathrm{m}$ global).

\begin{tabular}{c|c|c}
\hline Variável & Nível mínimo & Nível máximo \\
\hline Amina (EDAB) & $60 \mathrm{~g} / \mathrm{t}_{\text {SiO2 }}$ & $100 \mathrm{~g} / \mathrm{t}_{\text {SiO2 }}$ \\
\hline Amido (mandioca) & $500 \mathrm{~g} / \mathrm{t}_{\text {alim. }}$ & $1000 \mathrm{~g} / \mathrm{t}_{\text {alim. }}$ \\
\hline $\mathrm{pH}$ & 9,50 & 10,50 \\
\hline $\begin{array}{c}\text { Rotação durante } \\
\text { condicionamento }\end{array}$ & $1400 \mathrm{rpm}$ & $2000 \mathrm{rpm}$ \\
\hline
\end{tabular}

Tabela 2 - Níveis das variáveis (fração -150+45 $\mu \mathrm{m}$ ).

\begin{tabular}{c|c|c}
\hline Variável & Nível mínimo & Nível máximo \\
\hline Amina (EDAB) & $150 \mathrm{~g} / \mathrm{t}_{\mathrm{SiO} 2}$ & $250 \mathrm{~g} / \mathrm{t}_{\mathrm{SiO} 2}$ \\
\hline Amido (mandioca) & $500 \mathrm{~g} / \mathrm{t}_{\text {alim. }}$ & $1000 \mathrm{~g} / \mathrm{t}_{\mathrm{alim} .}$ \\
\hline $\mathrm{pH}$ & 9,50 & 10,50 \\
\hline $\begin{array}{c}\text { Rotação durante } \\
\text { condicionamento }\end{array}$ & $1400 \mathrm{rpm}$ & $2000 \mathrm{rpm}$ \\
\hline
\end{tabular}


Avaliação do efeito da granulometria no processo de flotação

O maior consumo de coletor exigido pela fração grossa $(-150+45 \mu \mathrm{m})$ deverá ser mais investigado, considerando outros aspectos, tais como: teores das frações granulométricas, maior tendência de consumo de coletor pela fração fina, em função da maior área superficial específica e distribuição granulométrica da polpa, que afeta a estabilidade da espuma.

A separação de frações granulométricas para o processamento em diferentes circuitos de flotação poderá trazer ganhos significativos para a indústria de minério de ferro, devido à ampla distribuição granulométrica processada nos atuais circuitos de flotação.

\section{Referências bibliográficas}

ANFRUNS, J.J., KITCHENER, J.A. The rate of capture of small particles in flotation. Trans. Inst. Min. Metal., v.86, p. C9-c15, 1977.

Tabela 3 - Níveis das variáveis (fração $-45 \mu \mathrm{m}$ ).

\begin{tabular}{c|c|c}
\hline Variável & Nível mínimo & Nível máximo \\
\hline Amina (EDAB) & $120 \mathrm{~g} / \mathrm{t}_{\mathrm{SiO} 2}$ & $200 \mathrm{~g} / \mathrm{t}_{\mathrm{SiO} 2}$ \\
\hline Amido (mandioca) & $500 \mathrm{~g} / \mathrm{t}_{\mathrm{alim} .}$ & $1000 \mathrm{~g} / \mathrm{t}_{\mathrm{alim} .}$ \\
\hline $\mathrm{pH}$ & 9,50 & 10,50 \\
\hline $\begin{array}{c}\text { Rotação durante } \\
\text { condicionamento }\end{array}$ & $1400 \mathrm{rpm}$ & $2000 \mathrm{rpm}$ \\
\hline
\end{tabular}

Tabela 4 - Distribuição granuloquímica da amostra deslamada.

\begin{tabular}{|c|c|c|c|c|}
\hline \multirow{2}{*}{$\begin{array}{l}\text { Malha } \\
(\mu \mathrm{m})\end{array}$} & \multicolumn{2}{|c|}{ \% Retido } & \multicolumn{2}{|c|}{ Análise Química (\%) } \\
\hline & Simples & Acumulado & $\mathrm{Fe}$ & $\mathrm{SiO}_{2}$ \\
\hline 150 & 3,00 & 3,00 & 33,70 & 47,54 \\
\hline 106 & 4,95 & 7,95 & 32,66 & 51,06 \\
\hline 75 & 7,30 & 15,25 & 28,78 & 57,53 \\
\hline 53 & 12,38 & 27,63 & 29,85 & 56,40 \\
\hline 45 & 11,67 & 39,30 & 25,11 & 62,90 \\
\hline-45 & 60,70 & 100,00 & 57,37 & 17,11 \\
\hline Global & \multicolumn{2}{|c|}{100,00} & 46,01 & 33,27 \\
\hline
\end{tabular}

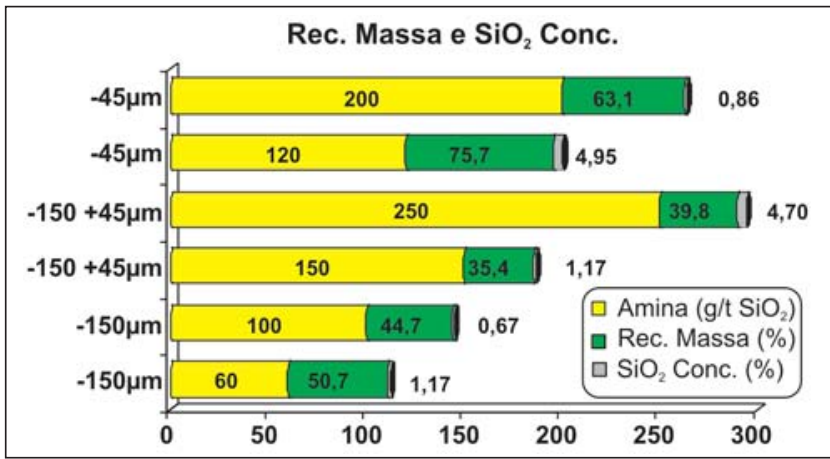

Figura 2 - Recuperação em massa e teor de $\mathrm{SiO}_{2}$ no concentrado para diferentes dosagens de amina.

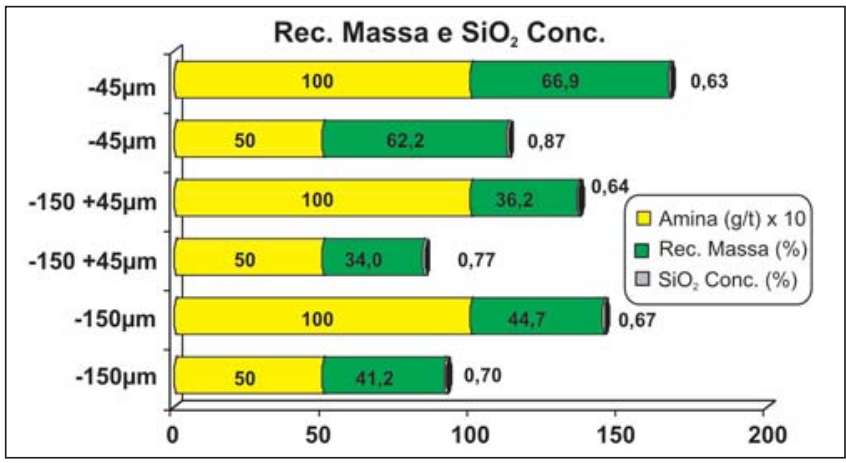

Figura 3 - Recuperação em massa e teor de $\mathrm{SiO}_{2}$ no concentrado para diferentes dosagens de amido.

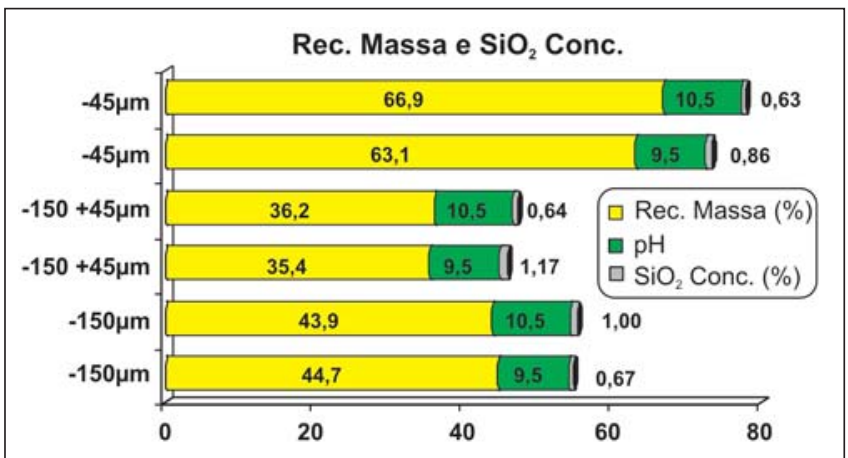

Figura 4 - Recuperação em massa e teor de $\mathrm{SiO}_{2}$ no concentrado para diferentes valores de $\mathrm{pH}$.

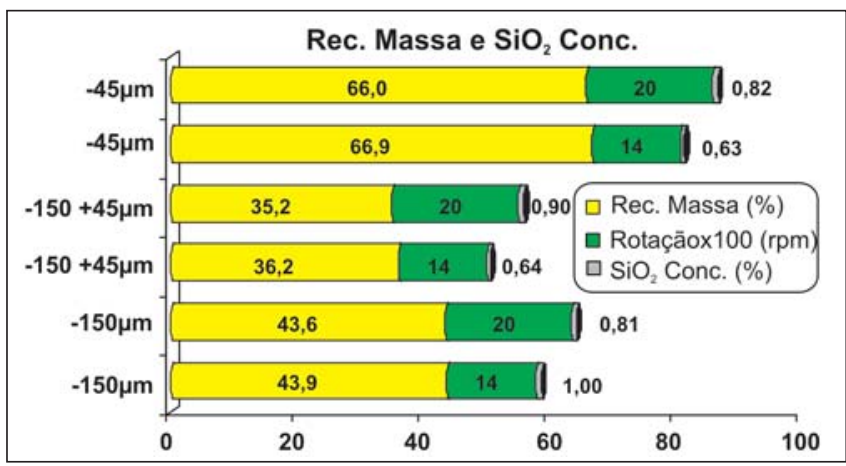

Figura 5 - Recuperação em massa e teor de $\mathrm{SiO}_{2}$ no concentrado para diferentes rotações durante condicionamento. 


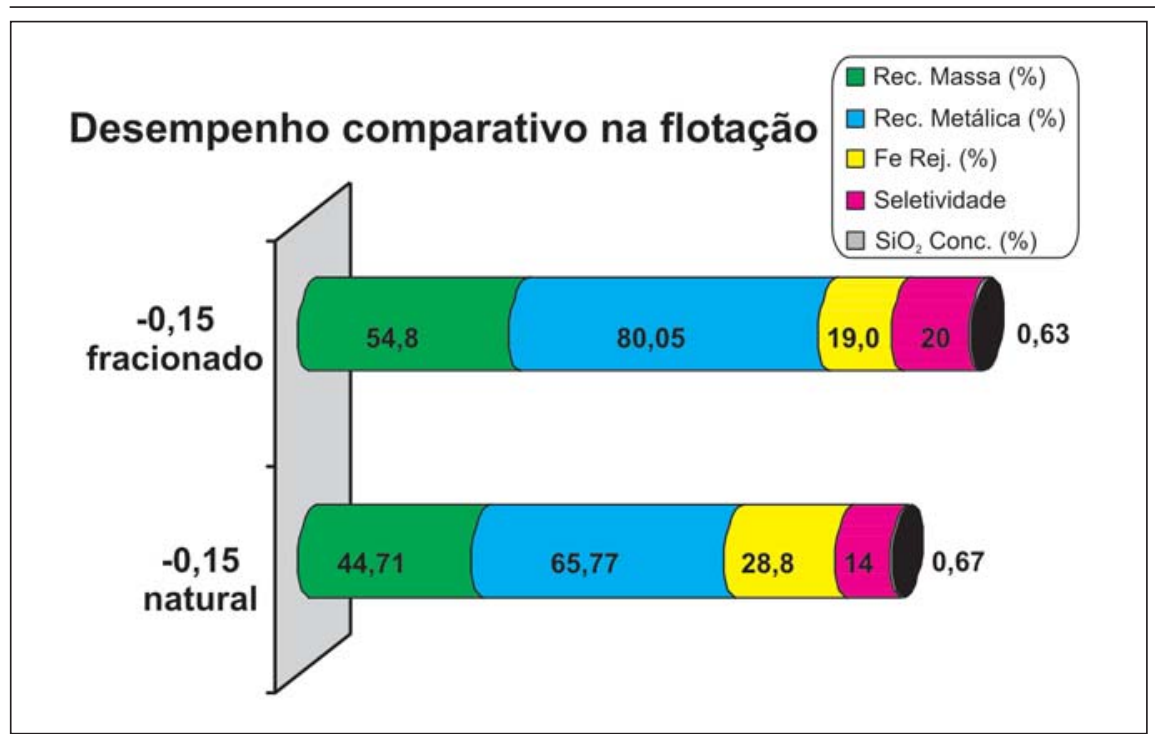

Figura 6 - Desempenho comparativo na flotação.

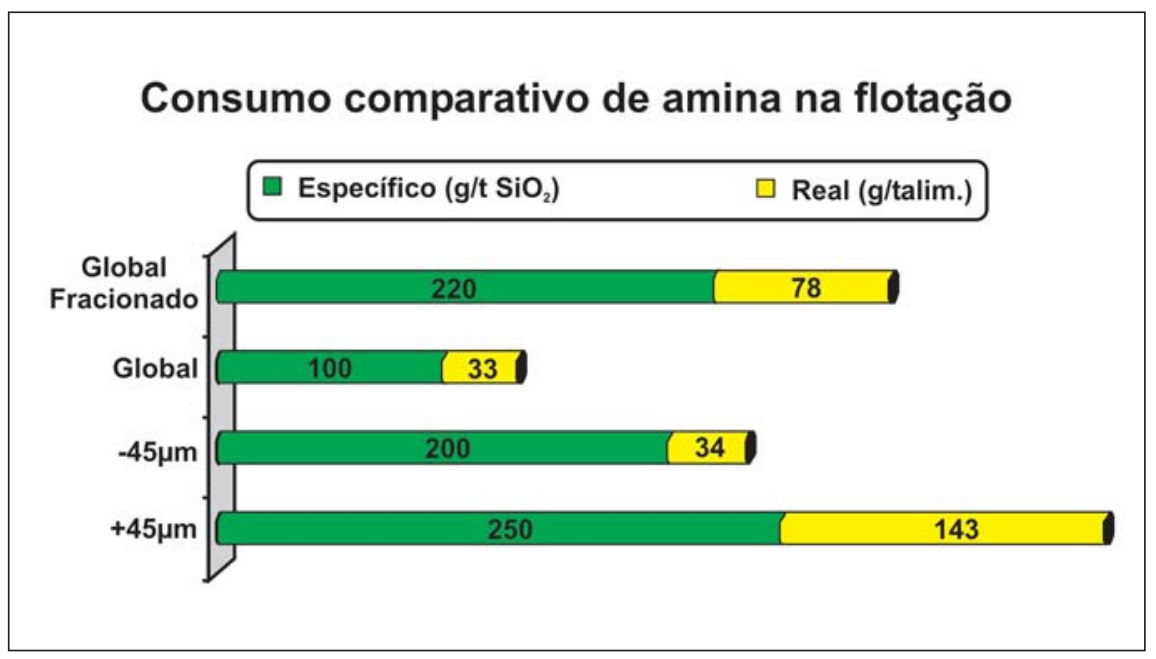

Figura 7 - Análise comparativa entre dosagens de coletores.

BRUM, I.S., RUBIO, J., VALDERRAMA,

L. Condicionamento turbulento para a recuperação de finos de minérios por flotação. In: ENCONTRO NACIONAL DE TRATAMENTO DE MINÉRIOS, v.1, p. 367-381, Rio de Janeiro, 3 a 6 de setembro de 1995.
BULATOVIC, S.M., SALTER, R.S. High intensity conditioning: a new approach to improve flotation of mineral slimes. In: Conference of Metallurgists, Halifax, Canadá, Proceedings... p.182-197, 1989. COLLINS, G. L., JAMESON, G.L. Experiments on the flotation of fine particles. The influence of particles size and charge. Chem. Eng. Sci., v.31, p. 985-991, 1976.

FLINT, L.R., HOWARTH, W.J. The collision efficiency of small particles with spherical air bublues. Chem. Eng. Sci., v.26, p. 1155-1168, 1971.

GLEMBOTSKY, V.A. Investigation of separate conditioning of sands and slimes with reagents prior to joint flotation. In: INTERNATIONAL MINERAL PROCESSING CONGRESS, 8, Paper S16, Leningrado, 1968.

GONZALES,G. Discussions and contributions. Trans. Int. Min. Metal., v. 87, p. c82-c83, 1978.

OLIVEIRA, J.F. Setor mineral: tendências tecnológicas. Texto elaborado para o projeto Setor Mineral - Tendências Tecnológicas, do Centro de Tecnologia Mineral - CETEM. 2006.

REAY, D., RATCLIFF, G.A. Removal of fine from water by dispersed air flotation: effects of bubble size and particle on collection efficiency. Can. J. of Chem. Eng., v.51, p.178-185, 1975.

ROBINSON, A. J. Relationship between particle size and collector concentration. Trans. Inst. Min. Metal., v.69, p. 45-62, 1975.

SCHULZE, H. I. Physico-chemical elementary processes in flotation. Amsterdam: Elsevier, 1984. v. 348. $238 \mathrm{p}$.

TOMLINSON, H.S., FLEMING, M.G. Flotation rate studies. In: INTERNATIONAL MINERAL PROCESSING CONGRESS, v.6, Proceedings, p. 563-579, Cannes, 1963.

TRAHAR, W.J. A rational interpretation of the role of particle size in flotation. Int. J. Miner. Process., v. 8, n. 4, p. 289327, 1981.

Artigo recebido em 22/04/2008 e aprovado em 11/06/2008.

\section{REM - Revista Escola de Minas www.rem.com.br}

